\title{
lonically cross-linked hyaluronic acid: wetting, lubrication, and viscoelasticity of a modified adhesion barrier gel
}

This article was published in the following Dove Press journal:

Medical Devices: Evidence and Research

15 December 2010

Number of times this article has been viewed

\author{
Katherine Vorvolakos' \\ Irada S Isayeva' \\ Hoan-My Do Luu' \\ Dinesh V Patwardhan' \\ Steven K Pollack ${ }^{2}$ \\ 'Division of Chemistry and Material \\ Science, ${ }^{2}$ Office of Science and \\ Engineering Laboratories, Center \\ for Devices and Radiological Health, \\ Food and Drug Administration, Silver \\ Spring, MD, USA
}

\begin{abstract}
Hyaluronic acid (HA), in linear or cross-linked form, is a common component of cosmetics, personal care products, combination medical products, and medical devices. In all cases, the ability of the HA solution or gel to wet surfaces and/or disrupt and lubricate interfaces is a limiting feature of its mechanism of action. We synthesized ferric ion-cross-linked networks of HA based on an adhesion barrier, varied the degree of cross-linking, and performed wetting goniometry, viscometry, and dynamic mechanical analysis. As cross-linking increases, so do contact angle, viscosity, storage modulus, and loss modulus; thus, wetting and lubrication are compromised. These findings have implications in medical device materials, such as adhesion barriers and mucosal drug delivery vehicles.
\end{abstract}

Keywords: hyaluron, adhesion barrier, wetting, contact angle, viscosity, lubrication, elasticity, viscoelastic, hydrogel, ferric

\section{Introduction}

Hyaluronic acid (HA) is a linear glycosaminoglycan polysaccharide composed of D-glucuronic acid and D- $N$-acetylglucosamine monomer unit. Its salt, shown in the left of reaction scheme in Figure 1, is ubiquitous in nature, ${ }^{1,2}$ it is water soluble, present in the intracellular matrix in all animals, and has been used in the replacement of synovial and intraocular fluids. Harvested from rooster combs, its molecular weight can range from 1 to 6 million daltons, with a polydispersity index (PDI) typically of $>1.3$. Bacterial fermentation can result in average molecular weights ranging from the thousands to the multiple millions. Aqueous solution viscosity increases with molecular weight and concentration and further increases lead to elastic character.

HA is used as a dietary supplement, an ingredient in topical moisturizers and gels and in medical devices, ${ }^{3}$ including but not limited to injectable fluids indicated for osteoarthritis ${ }^{4}$ and cosmetic dermal augmentation and rejuvenation, adjuncts to ophthalmic surgery, ${ }^{5}$ and adhesion barriers in abdominal surgery. ${ }^{6-8}$ Different applications require that HA adopt different degrees of cross-linking. These range from linear uncross-linked HA, employed in ophthalmic device materials, to crosslinked elastic hydrogels, as in structural implants. Intermediately, cross-linked viscoelastic fluids will be discussed in this article. Previous cross-linking efforts have included creating a physically, ionically, or covalently associated network of HA chains. For instance, Balazs and Leshchiner ${ }^{6}$ patented a method to cross-link HA and other molecules with divinyl sulfone. Carbodiimide has been used to cross-link $\mathrm{HA}$, as patented by Hamilton et $\mathrm{al}^{9}$ and explored by others, including Xuejun et $\mathrm{al}^{10}$ 


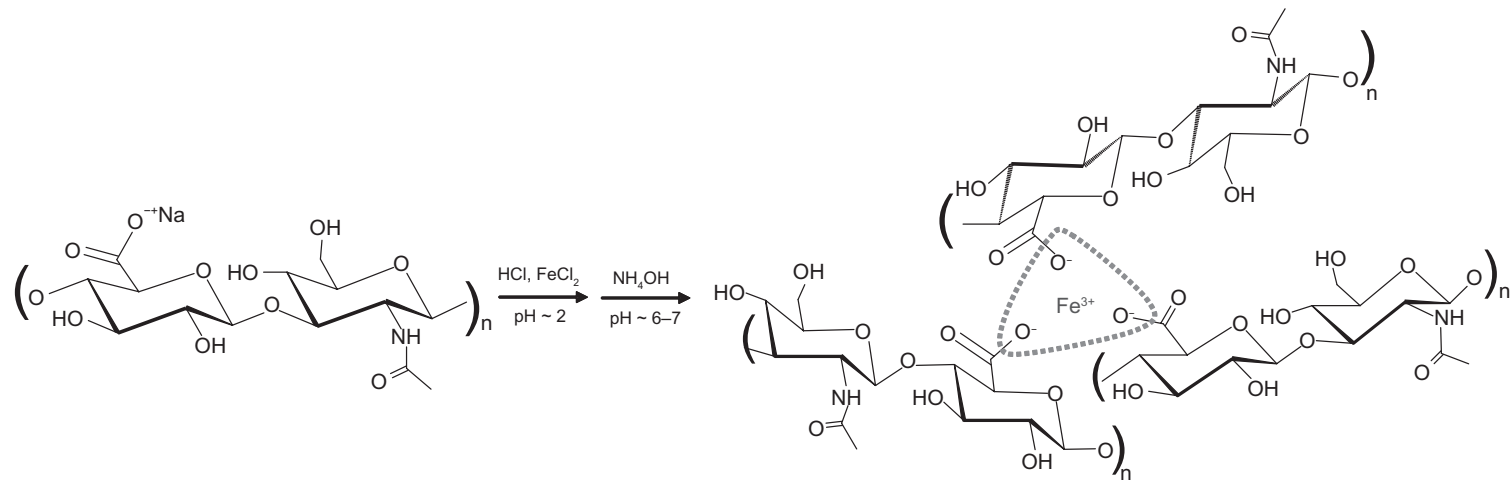

Figure I Reaction scheme and idealized structure of FeHA network.

and Tomihata and Ikada. ${ }^{11}$ Barbucci et a ${ }^{12}$ activated the carboxyl groups of HA and created a series of hydrogels using four diamine cross-linking agents. Multilayer films of HA with other biological polymers have been studied by Lavalle and colleagues. ${ }^{13,14}$ Šimkovic et al ${ }^{15}$ prepared several epichlorohydrin-cross-linked HA derivatives in the presence and absence of ammonium hydroxide. Mo et $a{ }^{16}$ reported the ability of calcium hyaluronate to cross-link when mixed with sodium-type gellan. Polyethylene glycol (PEG) derivatives have also served as cross-linkers; in 2000, Luo et al ${ }^{17}$ used PEG-propiondialdehyde to create an HA gel, whereas more recently, Segura et a $\mathrm{a}^{18}$ created a gel using a PEG diepoxide cross-linker. Della Valle and Romeo ${ }^{19}$ esterified the carboxyl groups of HA with alcohols to increase the relative number of hydrophobic to hydrophilic groups on the HA backbone, resulting in more robust mechanical properties. Mensitieri et $\mathrm{al}^{20}$ echoed this approach. Milella et $\mathrm{al}^{21}$ studied HA benzylic sponges for thermal and mechanical stability. Other examples of cross-linking abound in the literature, demonstrating the diversity of possibilities with this endogenous biopolymer. This work focuses on one such cross-linking possibility, as described below.

A patent of Huang et al $^{22}$ describes a method to ionically cross-link carboxyl-containing polysaccharides. One use of this method resulted in a network of HA incorporating trivalent ferric ions $\left(\mathrm{Fe}^{3+}\right)$ as cross-links, henceforth denoted as FeHA. According to the patent, ${ }^{8} 100 \%$ (as determined stoichiometrically) cross-linking implies that every carboxylate group on every HA chain should serve as a site for cross-linking, as idealized in Figure 1, with every trivalent ferric cation serving as cross-linker. It is wellknown since the work of Flory, ${ }^{23}$ however, that cross-link uptake slows and ceases as chains become immobilized; as a result only a fraction of the stoichiometrically predicted cross-linking occurs. Nevertheless, in this work, we will use stoichiometric cross-linking percentages to identify different FeHA materials.

A medical device based on this patent was used clinically as an abdominal adhesion barrier. ${ }^{6}$ Cross-linking was believed to delay metabolic clearance and allow the material to persist for the time window of adhesiogenesis, estimated to be within the first week to 10 days. During this period, FeHA was intended to provide a physical barrier between organs. For reasons not necessarily related to the present work, the device was eventually withdrawn from the market. We based our set of material compositions on this scientifically convenient patent recipe, which is not presently in use by the American medical device industry and is easily varied.

A medical device, by definition, has a mechanism of action largely independent of pharmacological chemical activity. A fluidic abdominal adhesion barrier device is therefore dependent on its physical properties to function. It must (1) disrupt the interface between surgically traumatized tissue surfaces by wetting one or both surfaces, (2) lubricate the interface, (3) resist displacement by the mechanical forces imposed by organs at rest or in motion, and (4) maintain forces native to a healthy presurgical site. As such, relevant properties include, respectively, (1) wetting, as indicated by contact angle $\theta$; (2) lubricity, as indicated by apparent viscosity $\eta ;$ (3) viscoelasticity, as indicated by elastic $\left(E^{\prime}\right)$ and viscous $\left(E^{\prime \prime}\right)$ moduli, measured using dynamic mechanical analysis (DMA); and (4) knowledge of how these compare to the native properties of abdominal organs and peritoneal fluid. The present work varies the nominal degree of crosslinking and examines the properties described above.

\section{Experimental methods Materials}

Unless stated otherwise, all chemicals were used as received. Medical grade sodium hyaluronate (NaHA) of molecular 
weight $=0.99 \mathrm{MDa}$ and $\mathrm{PDI}=1.3$ (Lifecore Biomedical Inc., Chaska, MN). The bacterial fermentation-produced NaHA (from Streptococcus equi sp) was preferred over animal-produced NaHA because of its high purity and low PDI. Iron (III) chloride hexahydrate, reagent grade EDTA, and 1.046 N HCl were purchased from Sigma-Aldrich (St Louis, MO). Sodium chloride in crystal form was purchased from Mallinckrodt (St Louis, MO). Sodium hydroxide in pellet form was purchased from EM Science (Gibbstown, NJ). Ammonium hydroxide, 28\% in solution, was purchased from Fluka, Sigma-Aldrich. All chemicals were used as received. A $2.5 \%$ (\% wt) $\mathrm{FeCl}_{3} \cdot 6 \mathrm{H}_{2} \mathrm{O}$ in $0.05 \mathrm{~N} \mathrm{HCl}$ solution was prepared by dissolving $0.8336 \mathrm{~g}$ of $\mathrm{FeCl}_{3} \cdot 6 \mathrm{H}_{2} \mathrm{O}$ with $32.49 \mathrm{~g}$ of $0.05 \mathrm{~N} \mathrm{HCl}$.

\section{Syntheses of FeHA gel networks}

The patent by Huang et $\mathrm{al}^{22}$ served as a starting point to prepare a FeHA network with nominally $90 \%$ stoichiometric cross-linking. All networks were prepared in clean, dry, $250 \mathrm{~mL}$ round-bottomed flasks; $0.3026 \mathrm{~g}$ of NaHA was dissolved in $57.66 \mathrm{~g}$ of deionized water (Milli-Q Plus; Millipore, Billerica, MA) and stirred gently until dissolved, resulting in $0.5 \% \mathrm{HA}$ solutions by mass. The solution $\mathrm{pH}$ was reduced to 2.0-2.5 via slow, stirred addition of $0.1 \mathrm{~N}$ $\mathrm{HCl}$ and estimated using $\mathrm{pH}$ paper. Upon $\mathrm{pH}$ stabilization, $\mathrm{FeCl}_{3}$ solution was added, resulting in a slight but discernible decrease in $\mathrm{pH}$. For nominal 50\%, 90\%, and 100\% cross-linking in FeHA gels (designated FeHA-50, FeHA-90, and FeHA-100), the molar $\left[\mathrm{Fe}^{3+}\right] /\left[\mathrm{COO}^{-}\right]$ratio was adapted to $0.165,0.3$, and 0.33 , respectively. The solution was stirred overnight. A $1.7 \mathrm{~N} \mathrm{NH}_{4} \mathrm{OH}$ solution was then added to neutralize the solution. If solution $\mathrm{pH}$ overshot to above 8 , it was adjusted using $\mathrm{HCl}$. The $\mathrm{pH}$ of the final FeHA networks was in the range of 6-7. The slight variation in added cross-linker solution resulted in mass concentrations of $0.005103,0.005011$, and 0.004989 , respectively, all of which were very slightly different from $0.5 \%$. Each preparation resulted in material that took the shape of its container and could be pipetted but appeared gel-like when gently shaken.

\section{Contact angle goniometry}

Static contact angle $(\theta)$ for each fluid, as illustrated in Figure 2, was measured at the National Institute of Standards and Technology Combinatorial Methods Center using a G2 contact angle measuring system (Kruss, Matthews, NC). Drops of the various FeHA gels were placed gently on a glass slide surface that had been exposed to low-level oxygen plasma in an Plasma Series SP100 Controller chamber (Anatech Ltd., Springfield, VA) for 1 minute. After removal from the plasma chamber, the slides were placed in clean polystyrene petri dishes and removed only when used for contact angle measurements. Immediately before the introduction of FeHA to a different area on the slide, water droplets exhibited negligible contact angles (ie, water flattened to a sheet) on each of the plasma-treated slides, indicating strong and consistent hydrophilicity, . The droplet size of the FeHA fluids was kept as close as possible to $1 \mu \mathrm{L}$. Evaporation was minimized by enclosing the goniometer stage and placing paper towels saturated with water in the enclosure. Contact angles were measured at approximately 1 and 5 minutes after placement to allow for transient drop relaxation. Three measurements per formulation were made. Static contact angles were measured for all three networks. Pendant drops of the fluids were suspended from the needle of the goniometer and brought slowly to the point contact with the glass. They were allowed to detach naturally from the needle to form spherical caps on the glass. All measurements were taken at $25^{\circ} \mathrm{C} \pm 2{ }^{\circ} \mathrm{C}$.

\section{Viscometry}

Shear viscosities of the FeHA-50, FeHA-90, and FeHA-100 networks were measured using an annular ring-G2 cone and plate viscometer (TA Instruments, New Castle, DE) in continuous mode. Measurements were made at $25^{\circ} \mathrm{C}$. Prior to the measurements, the viscometer was calibrated using Brookfield silicone oil standards. The FeHA networks were subjected to shear rates that varied from 0.1 to $100 \mathrm{~s}^{-1}$, and 10 data per decade were recorded. Averages and standard deviations of three measurements were calculated for each shear rate.

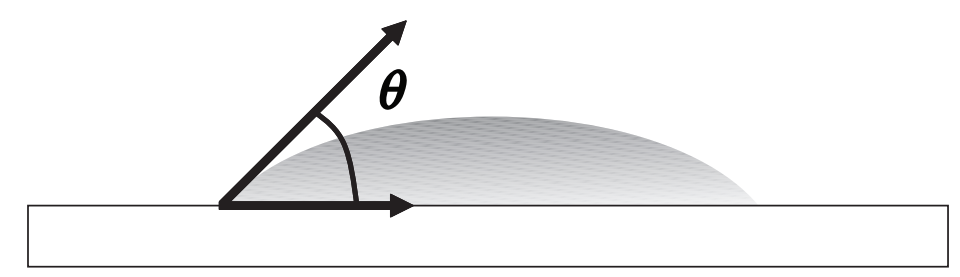

Figure 2 Contact angle $\theta$ is measured between the horizon and the tangent to the drop at the horizon. 


\section{Dynamic mechanical analysis}

DMA was carried out on FeHA-50, FeHA-90, and FeHA-100 networks using a Dynamic Mechanical Thermal Analyzer IV (Rheometric Scientific Inc., Piscataway, NJ) in parallel plate compression mode at $25^{\circ} \mathrm{C}$. Samples of FeHA network were placed on the lower plate, which was slowly raised to create a gap distance of $1 \mathrm{~mm}$ with the upper plate. Excess FeHA was removed from the perimeter of the round plates. Compression strain was $10 \%$ (well within the linear elastic regime, as determined previously from strain sweep measurements). Oscillatory compression took place over a frequency sweep $(0.1-100 \mathrm{~Hz})$; storage and loss moduli $\left(E^{\prime}\right.$ and $E^{\prime \prime}$, respectively) and phase angle ( $\tan \delta$ ) were measured. The average and standard deviation of three measurements were calculated for each data point.

\section{Results and discussion Contact angle goniometry}

The static contact angle as a function of nominal cross-linking is shown in Figure 3. Contact angle varied from as low as $\sim 7.5^{\circ}$ to as high as $\sim 17^{\circ}$ as cross-linking increased, indicating that the ability to wet a surface declines as cross-linking increases. The drops reached their terminal wetting within the first few seconds of placement; no difference was seen between contact angles measured at 1 and 5 minutes. To set the change in wettability in some practical context, we performed simple geometric calculations to determine the areal coverage per volume (ie, a wetting efficiency or "footprint") of $1 \mu \mathrm{L}$ spherical caps having the contact angles shown in

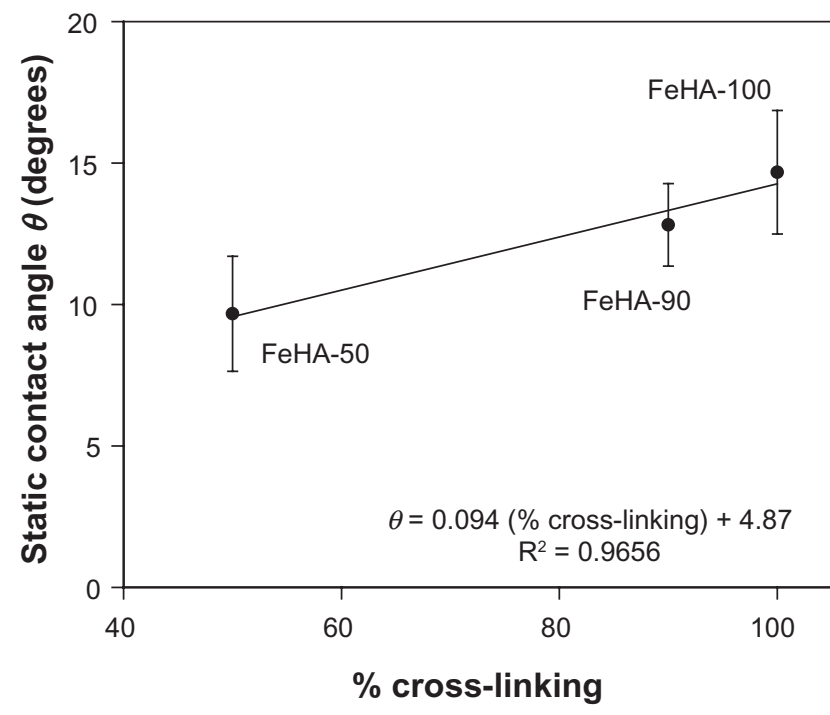

Figure 3 Static contact angle $\theta$ of ferric ion-cross-linked HA (FeHA) gels on clean oxidized glass as a function of percent cross-linking. Each data point represents a mean; error bars indicate standard deviation.
Figure 3. Figure 4 shows footprint as a function of crosslinking. As cross-linking increases from $50 \%$ to $100 \%$, the footprint reduces by as much as $40 \%$. This change in wetting is due to the change in reaction scheme. Although the effects of molecular weight on surface tension have long been studied for melts and solutions, ${ }^{24-27}$ the effect of partial cross-linking in HA gels has not been extensively studied. It is unclear whether the effect seen here is due to a difference in surface energetics, a difference in bulk properties, or some combination of both. For example, if cross-linking reduces the number of free ionic groups, the polarity of the FeHA surface may be thus reduced. Alternatively, bulk properties may complicate apparent interfacial energetics. We are presently conducting separate studies to explore the relationship between HA viscoelasticity ${ }^{28}$ and the dynamic wetting properties of uncross-linked HA solutions.

Regardless of the actual mechanism, these data demonstrate that the cross-linking can significantly affect the ability of a fluid to wet a surface. As such, cross-linking can alter the volume necessary for a device material or drug delivery vehicle to adequately cover a treatment area. An increase in material volume presents an additional burden to the body's mechanisms for degradation, metabolism, mechanical expulsion, or other mode of clearance. An inability to wet a surface automatically results in an inability to disrupt an interface. In the case of an abdominal adhesion barrier, an inability to disrupt the interface between organ surfaces precludes lubrication between them. The native lubricant between abdominal organs is peritoneal fluid, normally present in

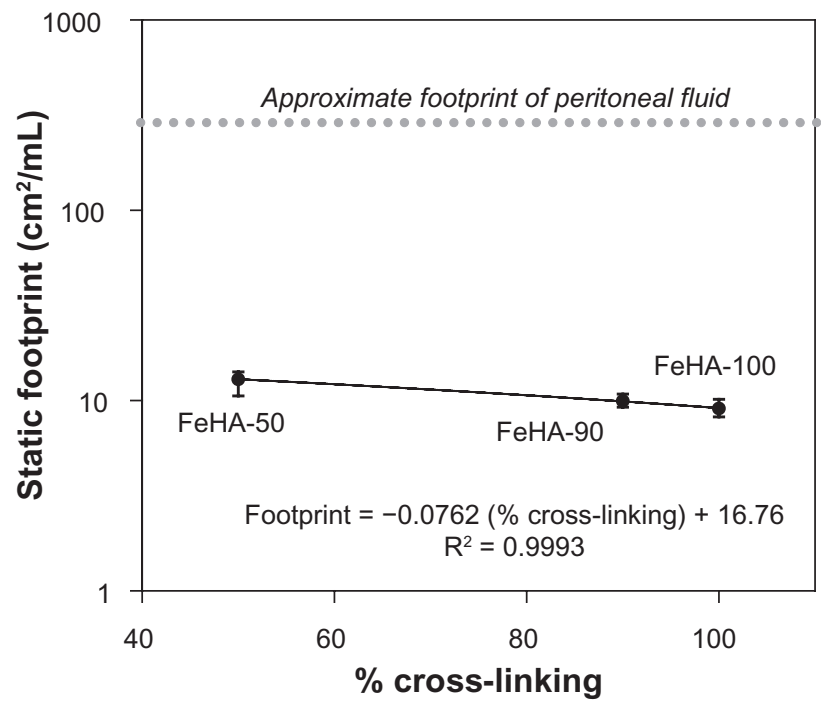

Figure 4 Wetting efficiency or "footprint" calculations based on the $\theta$ values as a function of cross-linking. Each data point represents a mean; error bars indicate standard deviation.

Abbreviation: FeHA, ferric ion-cross-linked HA. 
volumes in the order of $50 \mathrm{~mL}$, covering approximately $1.5 \mathrm{~m}^{2}$ of surface area, ${ }^{29}$ resulting in a characteristic footprint of approximately $285 \mathrm{~cm}^{2} / \mathrm{mL}$. Although the clean glass substrate used here is not directly comparable to abdominal tissue, its extreme hydrophilicity does present a "best-case" scenario for wetting by an aqueous solution. As such, it is clear that these FeHA gels would wet poorly compared with peritoneal fluid. Clinical implications are not obvious because a smaller footprint may only compromise, not necessarily prevent, the essential wetting. Further studies of the wettability of HA on specific tissues or mimics will serve to build understanding of how this ubiquitous biomolecule behaves.

The desired footprint may vary greatly with application and/or somatic site. However, the spatial presence of the material at surfaces and interfaces is only the first step to functioning. Once present, such materials may then be required to provide structural support, adhesive strength, or lubrication. The lubricating ability of the present FeHA networks is discussed below.

\section{Viscometry}

Soft polymeric materials used in medical devices are often charged with the task of lubricating organ interfaces. As such, viscometry represents a useful tool for performance evaluation. In the medical device industry, viscosity specifications are common product release criteria, which used as indication of product consistency. Therefore, it is useful to know how viscosity varies with reaction conditions. Figures 5, 6, and 7 show apparent viscosity $\eta$ as a function of shear rate for networks FeHA-50, FeHA-90, and FeHA-100, respectively. In all cases, shear thinning (a decrease in viscosity with increasing shear rate) is observed. In FeHA-50 and FeHA-90, increasing and decreasing the shear rate result in viscosity curves that nearly superimpose, indicating minimal hysteresis or degradation due to mechanical forces. In FeHA-100, there is a significant difference between the increasing and decreasing shear rate curves, indicating mechanical degradation. The following two general observations are made: (1) shear rate-dependent viscosity increases with increasing cross-linking and (2) the tendency to degrade upon exposure to mechanical forces increases with increasing cross-linking.

Peritoneal fluid, a natural lubricant of abdominal organs, has viscosities ${ }^{30}$ in the order of $10^{-3} \mathrm{~Pa}$ s. The fluids examined here display much higher viscosities, even in a shear rate range that assumes moderate interfacial sliding of

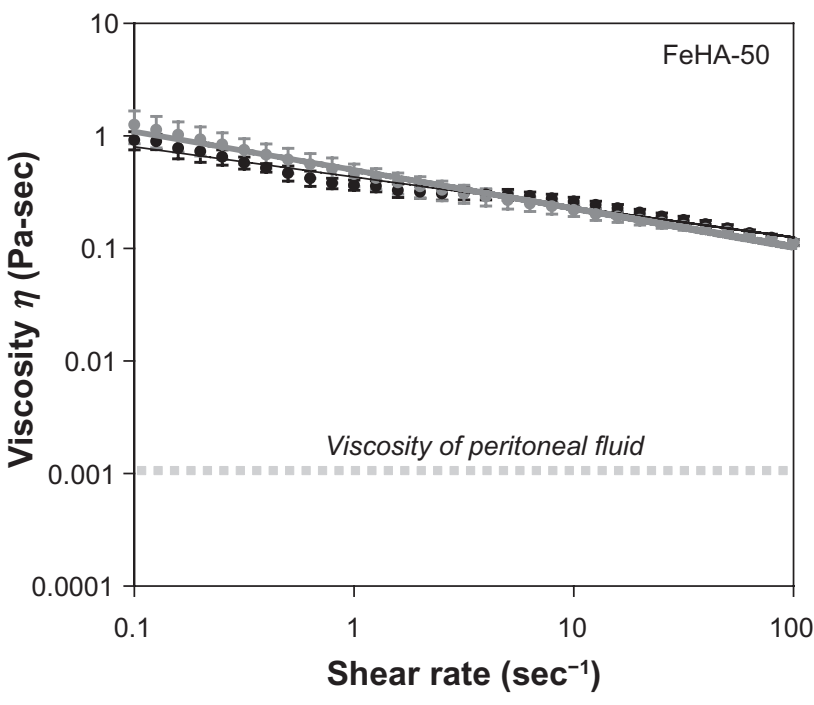

Figure 5 Viscosity as a function of increasing $(\bullet)$ and decreasing $(\bullet)$ shear rate for ferric ion-cross-linked HA (FeHA)-50 gel. The curves appear to superimpose with no notable hysteresis. The viscosity at all shear rates is orders of magnitude greater than that of peritoneal fluid. Each data point represents a mean; error bars indicate standard deviation.

$\operatorname{organs}^{31}\left(1-2 \mathrm{~s}^{-1}\right)$. In the peritoneum, lubrication is more complicated, aided by the specific biochemical species at the organ surfaces. Nevertheless, the higher apparent viscosity of the FeHA fluids implies that they would present non-native frictional forces to the peritoneum if introduced surgically. As discussed above, viscometry is only one part of a complete physical characterization; these fluidic materials are more complex.

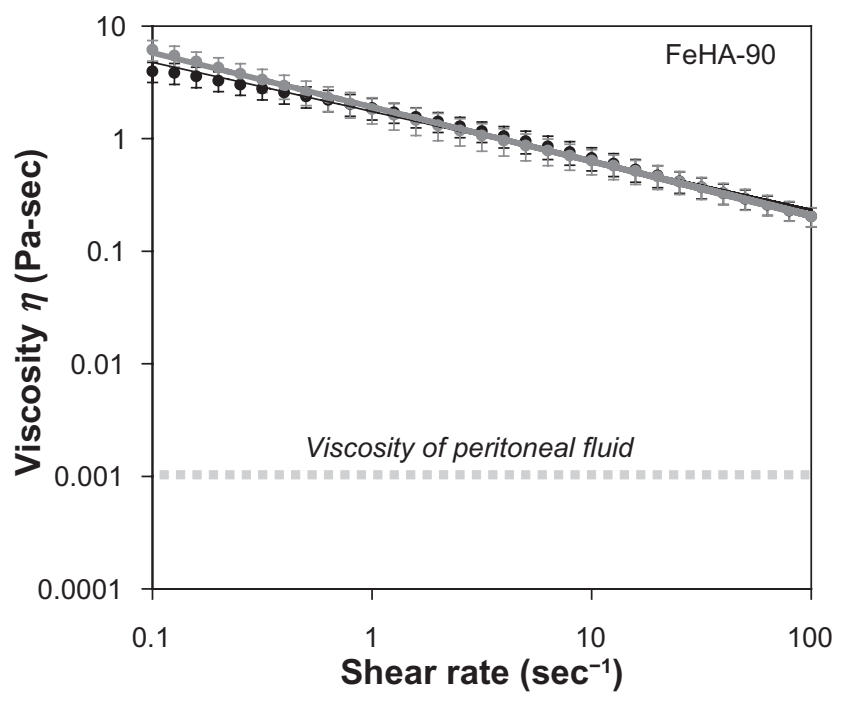

Figure 6 Viscosity as a function of increasing $(\bullet)$ and decreasing $(\bullet)$ shear rate for (a) ferric ion-cross-linked HA (FeHA)-90. The data are higher than for FeHA-50 but still show no hysteresis. The viscosity at all shear rates is orders of magnitude higher than that of peritoneal fluid. Each data point represents a mean; error bars indicate standard deviation. 


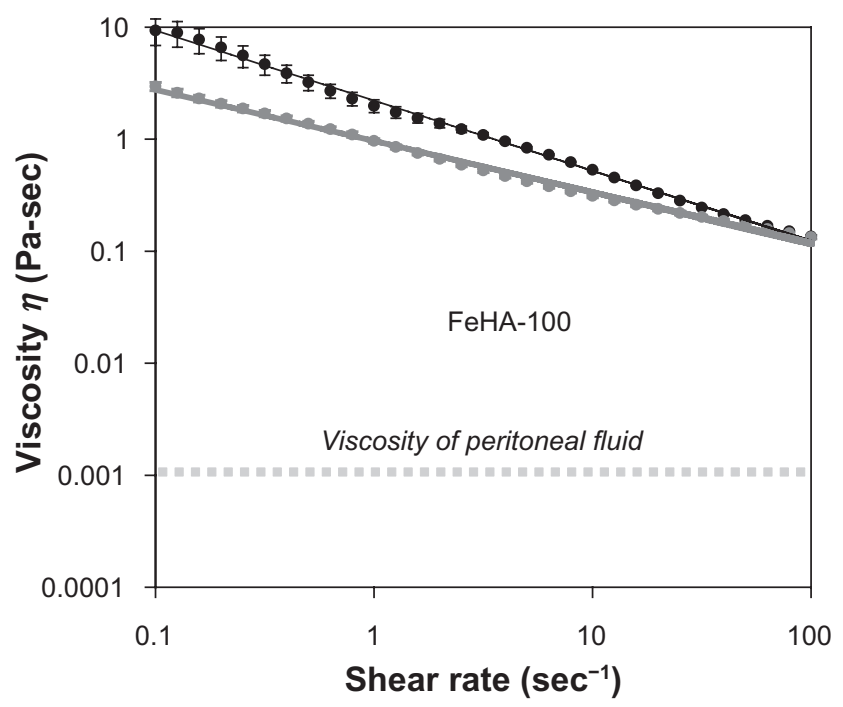

Figure 7 Viscosity as a function of increasing $(\bullet)$ and decreasing $(\bullet)$ shear rate for ferric ion-cross-linked HA (FeHA)-100. In addition to display higher viscosity, this gel displays hysteresis. The viscosity of the mechanically degraded material still exceeds that of peritoneal fluid by several orders of magnitude at all shear rates. Each data point represents a mean; error bars indicate standard deviation.

\section{Dynamic mechanical analysis}

As described previously, viscometry is a useful tool for characterizing mechanical properties of a fluidic material. Industry-wide, a viscosity specification is the most popular, and often the only, physical property release criterion for polymer solutions and flowing gels. Apparent viscosity, as reported below, may be used as an accurate predictive tool for how a lubricant will perform. However, viscometry is not a priori a complete tool for characterizing cross-linked materials. Viscometry assumes only dissipative character in a material, and does not distinguish between dissipative (viscous) and elastic (storage) characters. Although such resolution is unnecessary for materials that are purely viscous, it is required to describe the characteristics of viscoelastic materials, which may only be identified as such a posteriori. As mentioned in the introduction section, uncross-linked polymer solutions may exhibit some elastic characters, depending on molecular weight and concentration. Cross-linked aqueous systems are more likely to display viscoelasticity, which is quantified using DMA.

DMA was performed on FeHA-50, FeHA-90, and FeHA-100 in parallel plate compression mode. Figures 8, 9 , and 10 contain the frequency sweep data for each of the 3 networks, respectively; elastic modulus $E^{\prime}$, loss modulus $E^{\prime \prime}$, and phase shift $\tan \delta$ are shown for a frequency sweep of $0.1 \mathrm{~Hz}$ to approximately $100 \mathrm{~Hz}$. In all cases, $E^{\prime}$ exceeds $E^{\prime \prime}$ demonstrating that though the materials are fluidic, they have more elastic character than dissipative character and

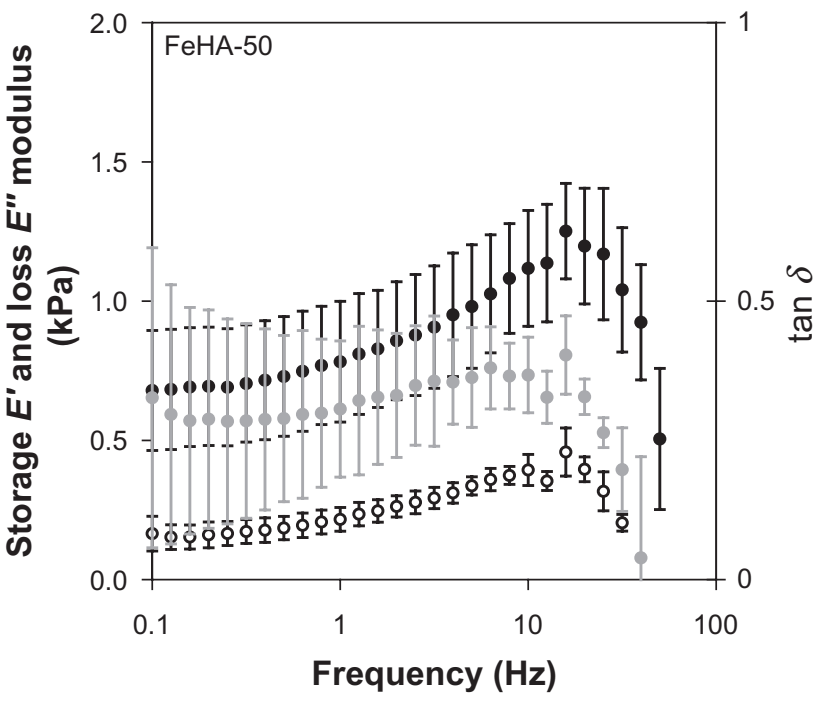

Figure 8 Storage modulus $\left(E^{\prime}, \bullet\right)$, loss modulus $\left(E^{\prime \prime}, \circ\right)$, and phase shift $(\tan \delta, \bullet)$ as a function of frequency for strain rate $\varepsilon=0.1$ for ferric ion-cross-linked HA (FeHA)-50. The material is a weak gel as indicated by $E^{\prime}$ exceeding $E^{\prime \prime}$ for all frequencies. Each data point represents a mean; error bars indicate standard deviation.

are therefore weak gels. Both $E^{\prime}$ and $E^{\prime \prime}$ increase as a function of frequency until immediately above $10 \mathrm{~Hz}$, at which point they peak and then decrease drastically. The peak frequency is independent of cross-linking percentage for both $E^{\prime}$ and $E^{\prime \prime}$. It was noted that after each frequency sweep (ie, several seconds after motion stopped), the networks appeared more liquid-like when removed from between the parallel plates, indicating mechanical degradation, possibly due to the breaking of ionic cross-links. These data demonstrate

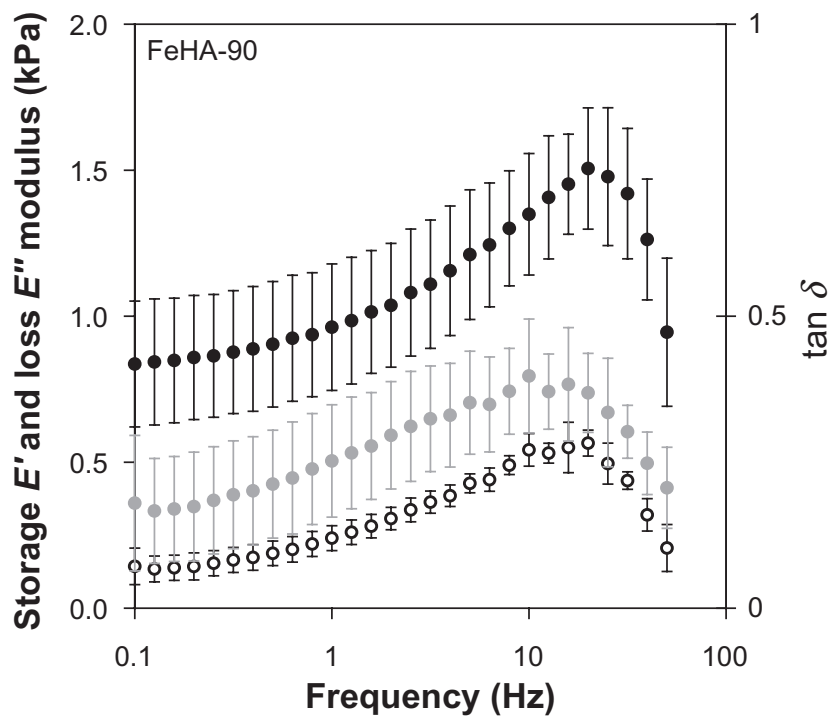

Figure 9 Storage modulus $\left(E^{\prime}, \bullet\right)$, loss modulus $\left(E^{\prime \prime}, \circ\right)$, and phase shift $(\tan \delta, \bullet)$ as a function of frequency for strain rate $\varepsilon=0.1$ for ferric ion-cross-linked HA (FeHA)-90. The material is a weak gel as indicated by $E^{\prime}$ exceeding $E^{\prime \prime}$ for all frequencies. $E^{\prime}$ of FeHA-90 exceeds that of FeHA-50, but $E^{\prime \prime}$ appears similar. Each data point represents a mean; error bars indicate standard deviation. 


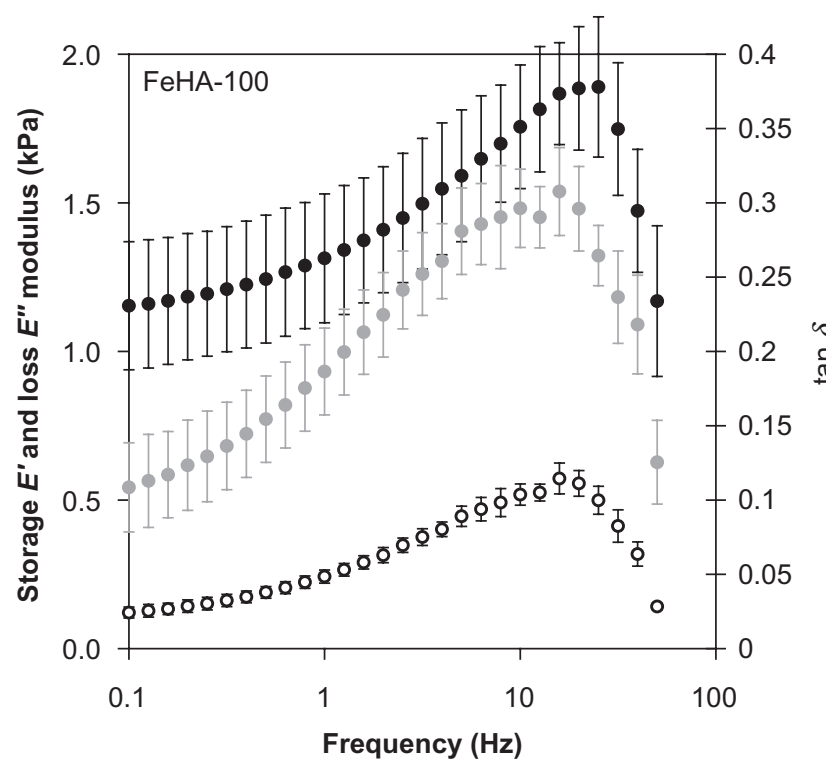

Figure 10 Storage modulus $\left(E^{\prime}, \bullet\right)$, loss modulus $\left(E^{\prime \prime}, \circ\right)$, and phase shift $(\tan \delta, \bullet)$ as a function of frequency for strain rate $\varepsilon=0.1$ for ferric ion-cross-linked HA (FeHA)-100. The material is a weak gel as indicated by $E^{\prime}$ exceeding $E^{\prime \prime}$ for all frequencies. $E^{\prime}$ of FeHA- 100 exceeds that of FeHA-90 and FeHA-50, but $E^{\prime \prime}$ appears similar in all cases. Each data point represents a mean; error bars indicate standard deviation.

that viscometry is a scientifically insufficient method of characterization.

The range of perturbation rate that is most relevant in material characterization depends, like footprint, on the application and deployment site. In the case of an abdominal adhesion barrier, frequencies in the order of $1 \mathrm{~Hz}$ are relevant, corresponding to walking, peristalsis, diaphragmatic expansion-contraction cycle, pulse rate, and other voluntary and involuntary motions. Figure 11 shows that at $1 \mathrm{~Hz}$, the elastic modulus $E^{\prime}$ ranges from approximately $0.6-1.4 \mathrm{kPa}$, and viscous modulus $E^{\prime \prime}$ varies only slightly around $0.25 \mathrm{kPa}$. Recorded values for elastic moduli of peritoneal organs $\mathrm{s}^{32,33}$ are in the range of 1-10 $\mathrm{MPa}$, much higher than those of the FeHA gels. This huge difference means that the FeHA gels would not present compressive mechanical forces exceeding normal ones in the peritoneum (unlike the viscometry data, which indicate that friction would increase). However, it also means that the FeHA gels would be unlikely to resist displacement and would be squeezed out between two organs. It is important to recall that the present measurements were made at $25^{\circ} \mathrm{C}$ while organs function at $37^{\circ} \mathrm{C}$. Actual measurements at $37^{\circ} \mathrm{C}$ would be valuable to further resolve differences between organs and implant. However, even in the case of an unlikely large percentage increase of the elastic modulus, we do not expect the prediction about relative compressive forces to change.

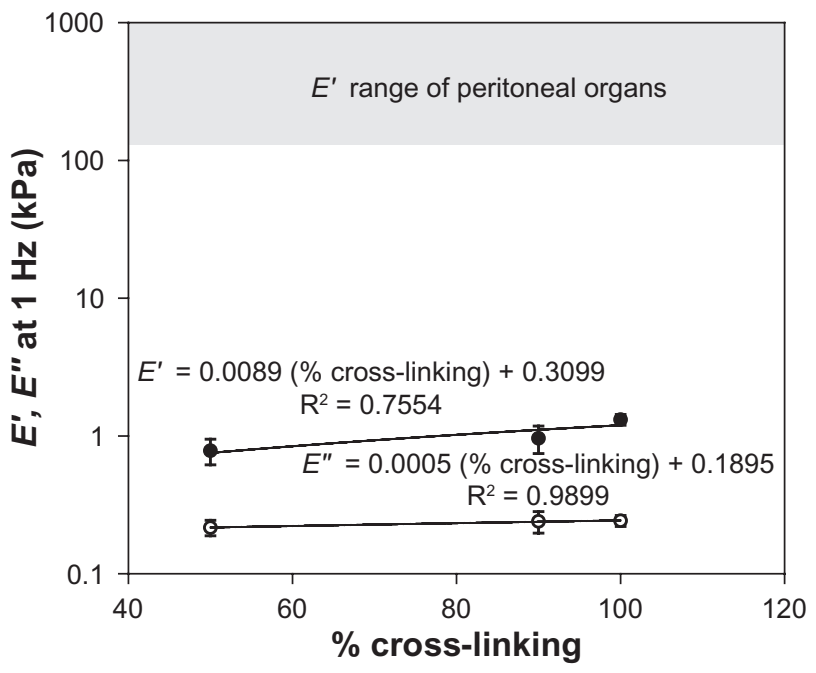

Figure II Storage $\left(E^{\prime}, \bullet\right)$ and loss $\left(E^{\prime \prime}, \circ\right)$ moduli at the physiologically relevant perturbation frequency of $\mathrm{I} \mathrm{Hz}$ as a function of percent cross-linking. Although the elastic modulus varies with cross-linking, it does not approach the modulus range of peritoneal organs. Loss modulus does not change significantly with cross-linking. Each data point represents a mean; error bars indicate standard deviation.

The physical properties of the FeHA networks measured here are determined by the reaction chemistry, such as precross-linking chain conformation, amount and accessibility of cross-linker to reactive carboxylate groups, $\mathrm{pH}$, and ionic strength. In this work, only cross-link amount was varied. The reaction $\mathrm{pH}$ was kept as close as possible to 2.5. At $\mathrm{pH} 2.5$, the relatively high viscosity of uncross-linked HA solutions has been attributed to stiffness of individual chains due to a critical balance between attractive (H-bonding) and repulsive (anionic) forces and later to interchain association as evidenced by a minimum in its self-diffusion coefficient ${ }^{34}$ and a maximum in tracer diffusion. These two attributes are mutually supportive, and it is intuitive that both stiffness and association are more likely to increase with expanded chains. At $\mathrm{pH}$ 2.5, spatial accessibility of the cross-link ferric ions to the carboxylate sites is also relatively maximized. The effect of reaction $\mathrm{pH}$ on the properties of other variations of FeHA networks is investigated by Isayeva et $\mathrm{al}^{31}$ who found that reaction $\mathrm{pH}$ can affect final lubrication properties and homogeneity, even if final $\mathrm{pH}$ is maintained constant. Such work is important because during manufacturing, medical device materials undergo fluctuations in $\mathrm{pH}$, ionic strength, and mechanical forces and the sensitivity of safety and performance properties, including homogeneity ${ }^{35-37}$, to these fluctuations is often overlooked.

The slight increase in contact angle with increased cross-linking and significant decrease in footprint demonstrates how easily wetting is affected. The more complicated questions naturally follow because the disruption of an 
interface by a fluid depends not only on the fluid but also on the two surfaces involved; these may be hydrophilic, hydrophobic, lipophilic, lipophobic, or any temporal or spatial combination thereof. In vitro performance testing should consider not only the properties of the device material or drug delivery medium but also the inherent surface properties of the target site.

In addition to the static properties of the interface, motions and forces are relevant. In the present case, a decrease in wettability with increased cross-linking is accompanied by an increase in apparent viscosity, ie, a decrease in lubricity over the entire shear rate range examined. Not only is wetting access restricted but also lubrication is poorer. In the relevant shear rate range (1-2 s ${ }^{-1}$ ), all networks exceeded the viscosity of peritoneal fluid. As such, abdominal organs would experience greater friction than the friction familiar to a healthy site. The relevant shear rate range and desired viscosity for other somatic sites and situations are naturally expected to vary. (For a contrasting example, when a person blows his/her nose, shear rates of $\sim 1,500 \mathrm{~s}^{-1}$ are generated; 38 material deployed nasally must perform under drastically different conditions than that deployed to the peritoneum). Fluid viscosities also vary over several orders of magnitude in the body; synovial fluid, eg, exhibits viscosity millions of times greater than peritoneal fluid. Orthopedic application designs of hydrogels should consider such facts.

Although viscometry is useful as a predictor of lubricity, it does not account for the elastic character of a material. DMA has shown that the elastic component of these networks is higher than the viscous one. It is beyond the scope of this work to discuss the effect that mechanical forces may have on adhesiogenesis. However, the preservation of native mechanical properties at sites of medical intervention is intuitive, as it helps to maintain healthy mechanotransduction, ${ }^{38-55}$ cellular communication that uses tactile information to effect and affect biochemical signals. Mechanical properties play a nontrivial role in the cause and manifestation of many diseases and medical events. For example, myocardial infarctions, scleroderma, Cushing disorder, initial cancer detection, and other phenomena all involve deviations from healthy mechanical properties and forces. Obviously, tissue sites would have characteristic force signatures; the moduli of organs vary over several orders of magnitude and exhibit complex patterns on various length scales. In addition to mechanical property variations, operative frequencies differ with application and deployment site. To complicate matters further, operative frequency of a gel device may depend not only on the native organ frequency but also on an adjunct device. In ophthalmic surgery to remove a cataract, eg, uncross-linked HA solutions are subjected to perturbation rates reaching $50 \mathrm{kHz}$ by phacoemulsification tools. The response of device and drug delivery materials to physiological and other operative frequencies is critical to acute long-term safety and performance.

The interplay between appropriate specifications for device or combination product materials presents an additional challenge to design. Chemical design and manufacturing that focus on lubricity or mechanical properties as parameters could compromise wetting, or vice-versa. Direct design accountability to all relevant parameters should be maintained.

\section{Summary}

In this limited study of FeHA networks, we have demonstrated the sensitivity of contact angle, apparent viscosity, and mechanical properties to cross-linking. We have shown that cross-linking can compromise wetting and lubrication and can produce materials with mechanical properties softer than or comparable to abdominal organs. We verified that viscometry, commonly used to characterize polymer solutions and hydrogels, has some utility in predicting lubricity; however, it is one part of a more complete characterization because it assumes only dissipative character. Viscoelasticity should be properly characterized and design specifications should be clinically justified.

The chemical and physical properties of device materials and drug delivery media have obvious clinical implications, or they would not be used. We echo here that it is intuitive for a device material or drug delivery medium to be least disruptive to the body if it preserved the wetting, lubrication, and mechanical properties native to the site of intervention. The characteristic footprint and operative shear rates and perturbation frequencies of phenomena, whether physiological (eg, diaphragmatic motion) or clinically induced (eg, phacoemulsification), are paramount in optimal design, development, and manufacture of such soft, complex materials.

\section{Disclosure}

Statements in this manuscript reflect the opinions of the authors and do not necessary reflect the opinions of the US Food and Drug Administration. The mention of commercial products, their source, or their use in connection with the material reported herein is not to be construed as either an 
actual or implied endorsement by the US Food and Drug Administration.

\section{References}

1. Lapčík L Jr, Lapčík L, de Smedt S, Demeester J, Chabreček P. Hyaluronan: preparation, structure, properties, and applications. Chem Rev. 1998;98:2663-2683.

2. Lee JY, Spicer AP. Hyaluronan: a multifunctional, megaDalton, stealth molecule. Curr Opin Cell Biol. 2000;12:581-586.

3. US Food and Drug Administration. Device advice: Device regulation and guidance. 2010. Available from: http://www.fda.gov/cdrh/ devadvice/312.html. Accessed Sept 20, 2010.

4. Barbucci R, Lamponi S, Borzacchiello A, et al. Hyaluronic acid hydrogel in the treatment of osteoarthritis. Biomaterials. 2002;23: 4503-4513.

5. Arshinoff SA, Jafari M. Laboratory science: new classification of ophthalmic viscosurgical devices. J Cataract Refract Surg. 2005;23: 2167-2171.

6. Balazs EA, Leshchiner A. Cross-linked gels of hyaluronic acid and products containing such gels. US patent 4,582,865; 1986.

7. Thornton MH, Johns DB, Campeau JD, Hoehler F, DiZerega GS. Clinical evaluation of $0.5 \%$ ferric hyaluronate adhesion prevention gel for the reduction of adhesions following peritoneal cavity surgery: open label pilot study. Hum Reprod. 1998;13(6):1480-1485.

8. Yeo Y, Adil M, Bellas E, Astashkina A, Chaudhary N, Kohane DS. Prevention of peritoneal adhesions with an in situ cross-linkable hyaluronan hydrogel delivering budesonide. J Control Release. 2007; 120:178-185.

9. Hamilton R, Fox EM, Acharya RA, Walts AE. Water insoluble derivatives of hyaluronic acid. US patent 4,937,270. 1990.

10. Xuejun X, Netti PA, Ambrosio L, Nicolais L, Sannino A. Preparation and characterization of hydrogel from low-molecular weight hyaluronic acid. J Bioact Compat Polym. 2004;19:5-15.

11. Tomihata K, Ikada Y. Crosslinking of hyaluronic acid with water-soluble carbodiimide. J Biomed Mater Res. 1997;37:243-251.

12. Barbucci R, Rappuoli R, Borzacchiello A, Ambrosio L. Synthesis, chemical and rheological characterization of new hyaluronic acid-based hydrogels. J Biomater Sci Polym Ed. 2000;11(4):383-399.

13. Richert L, Lavalle P, Payan E, et al. Layer by layer buildup of polysaccharide films: physical chemistry and cellular adhesion aspects. Langmuir. 2004;20(2):448-458.

14. Collin D, Lavalle P, Méndez Garza J, Voegel JC, Schaaf P, Martinoty P. Mechanical properties of cross-linked hyaluronic acid/poly-(L-lysine) multilayer films. Macromolecules. 2004;37:10195-10198.

15. Šimkovic I, Hricovíni M, Šoltés L, Mendichi R, Cosentino C. Preparation of water-soluble/insoluble derivatives of hyaluronic acid by cross-linking with epichlorohydrin in aqueous $\mathrm{NaOH} / \mathrm{NH}_{4} \mathrm{OH}$ solution. Carbohydr Polym. 2000;41:9-14.

16. Mo Y, Kubota K, Nishinari K. Rheological evidence of the gelation behavior of hyaluronan-gellan mixtures. Biorheology. 2000;37: 401-408.

17. Luo Y, Kirker K, Prestwich G. Crosslinked hyaluronic acid hydrogel films: new biomaterials for drug delivery. J Control Release. 2000;69; 169-184.

18. Segura T, Anderson BC, Chung PH, Webber RE, Shull KR, Shea LD. Crosslinked hyaluronic acid hydrogels: a strategy to functionalize and pattern. Biomaterials. 2005;26:359-371.

19. Della Valle F, Romeo A. New polysaccharide esters and their salts. European patent 216453; 1987.

20. Mensitieri M, Ambrosio L, Nicolais L, Bellini D, O’Regan M. Viscoelastic properties modulation of a novel autocrosslinked hyaluronic acid polymer. J Mater Sci Mater Med. 1996;7(11):695-698.

21. Milella E, Brescia E, Massaro C, Ramires PA. Chemico-physical properties of hyaluronan-based sponges. J Biomed Mater Res. 2000; 52:695-700.

22. Huang WJ, Johns DB, Kronenthal RL. Ionically crosslinked carboxylcontaining polysaccharides for adhesion prevention. US patent $5,532,221 ; 1996$.
23. Flory PJ. Molecular size distribution in three dimensional polymers; Part II; trifunctional branching units. J Am Chem Soc. 1941;63(11): 3091-3096.

24. LeGrand DC, Gaines GL Jr. The molecular weight dependence of polymer surface tension. J Colloid Interface Sci. 1969;31(2):162-167.

25. Chang SA, Gray DG. The surface tension of aqueous hydroxypropyl cellulose solutions. J Colloid Interface Sci. 1978;67(2):255-265.

26. Persson B, Nilsson S, Bergman R. Dynamic surface tension of dilute aqueous solutions of nonionic cellulose derivatives in relation to other macromolecular characterization parameters. J Colloid Interface Sci. 1999;218:433-441.

27. Qun G, Ajun W. Effects of molecular weight, degree of acetylation and ionic strength on surface tension of chitosan in dilute solution. Carbohydr Polym. 2006;64:29-36.

28. Kobayashi Y, Okamoto A, Nishinari K. Viscoelasticity of hyaluronic acid with different molecular weights. Biorheology. 1994;31(3): $235-244$.

29. Albanese AM, Albanese EF, Miño JH, Gomez E, Zandomeni M, Merlo AB. Peritoneal surface area: measurements of 40 structures covered by the peritoneum: correlation between total peritoneal surface area and the surface calculated by formulas. Surg Radiol Anat. 2009; 31(5):369-377.

30. Ott DE. Laparoscopy and tribology: the effect of laparoscopic gas on peritoneal fluid. J Am Assoc Gynecol Laparosc. 2001;8:117-123.

31. Isayeva I, Das SS, Chang A, et al. pH effect on the synthesis, shear properties and homogeneity of iron-crosslinked hyaluronic acidbased gel/adhesion barrier. J Biomed Mater Res B Appl Biomater. 2010;95(1):9-18.

32. Roeder R, Wolfe J, Lianakis N, Hinson T, Geddes LA, Obermiller J. Compliance, elastic modulus and burst pressure of small-intestine submucosa (SIS), small diameter vascular grafts. J Biomed Mater Res. 1999;47(1):65-70

33. Carter FJ, Frank TG, Davies PJ, McLean D, Cuschieri A. Measurements and modeling of the compliance of human and porcine organs. Med Image Anal. 2001;5:231-236.

34. Hardingham T. Solution properties of hyaluronan. In: Garg HG, Hales CA, editors. Chemistry and Biology of Hyaluronan. Oxford, UK: Elsevier Ltd; 2004:chap 1.

35. Mercê ALR, Carrere LCM, Romanholi LKS, Recio MAL. Aqueous and solid complexes of iron(III) with hyaluronic acid potentiometric titrations and infrared spectroscopy studies. J Inorg Biochem. 2002;89: 212-218.

36. Sipos P, St. Pierre TG, Tombacz E, Webb J. Rod-like iron(III) oxyhydroxide particles in iron(III)-polysaccharide solutions. J Inorg Biochem. 1995;58:129-138.

37. Sipos P, Berkesi O, Tombacz E, St. Pierre TG, Webb J. Formation of spherical iron(III) oxyhydroxide nanoparticles sterically stabilized by chitosan in aqueous solutions. J Inorg Biochem. 2003:95;55-63.

38. Gwaltney JM Jr, Hendley JO, Phillips CD, Bass CR, Mygind N, Winther B. Nose blowing propels nasal fluid into the paranasal sinuses. Clin Infect Dis. 2000;30:387-391.

39. Huang $\mathrm{S}$, Ingber DE. The structural and mechanical complexity of cell-growth control. Nat Cell Biol. 1999;1:E131-E138.

40. Ingber DE. Tensegrity I. Cell structure and hierarchical systems biology. J Cell Sci. 2003;116(7):1157-1173.

41. Ingber DE. Tensegrity II. How structural networks influence cellular information processing networks. J Cell Sci. 2003;116(8): 1397-1408.

42. Huang S, Ingber D. Cell tension, matrix mechanics, and cancer development. Cancer Cell. 2005;8(3):175-176.

43. Kwon RY, Jacobs CR. Time-dependent deformations in bone cells exposed to fluid flow in vitro: investigating the role of cellular deformation in fluid flow-induced signaling. J Biomech. 2007;40:3162-3168.

44. Shieh AC, Athanasiou KA. Dynamic compression of single cells. Osteoarthr and Cartil. 2007;15:328-334.

45. Helmke B, Schwartz M. Putting the squeeze on mechanotransduction. Dev Cell. 2004;6(6):745-746. 
46. Chatzizisis Y, Coskun AU, Jonas M, Edelman ER, Feldman CL, Stone PH. Role of endothelial shear stress in the natural history of coronary atherosclerosis and vascular remodeling. J Am Coll Cardiol. 2007;49(25):2379-2393.

47. Lee SE, Kamm RD, Mofrad MRK. Force-induced activation of talin and its possible role in focal adhesion mechanotransduction. J Biomech. 2007;40:2096-2106.

48. Darling EM, Topel M, Zauscher S, Vail TP, Guilak F. Viscoelastic properties of human mesenchymally-derived stem cells and primary osteoblasts, chondrocytes, and adipocytes. J Biomech. 2008;41(2): 454-464.

49. Ko KS, McCulloch CAG. Intercellular mechanotransduction: cellular circuits that coordinate tissue responses to mechanical loading. Biochem Biophys Res Commun. 2003;285:1077-1083.

50. Qin X, Tian J, Zhang P, Fan Y, et al. Laminar shear stress up-regulates the expression of stearoyl-CoA desaturase-1 in vascular endothelial cells. Cardiovas Res. 2007;74:506-514.
51. Schwarz US, Bishofs IB. Physical determinants of cell organization in soft media. Med Eng Phys. 2005;27:763-772.

52. Agar A, Li S, Agarwal N, Coroneo MT, Hill MA. Retinal ganglion cell line apoptosis induced by hydrostatic pressure. Brain Res. 2006;1086: 191-200.

53. Ghosh K, Pan Z, Guan E, Ge S, et al. Cell adaptation to a physiologically relevant ECM mimic with different viscoelastic properties. Biomaterials. 2007;28:671-679.

54. Selwyn DA, Raphael JH, Lambert DG, Langton JA. Effects of morphine on human nasal cilia beat frequency in vitro. Br J Anaesth. 1996;76: 274-277.

55. Girard PP, Cavalcanti-Adam EA, Kemkemer R, Spatz JP. Cellular chemomechanics at interfaces: sensing, integration and response. Soft Matter. 2007;3:307-326.

\section{Publish your work in this journal}

Medical Devices: Evidence and Research is an international, peerreviewed, open access journal that focuses on the evidence, technology, research, and expert opinion supporting the use and application of medical devices in the diagnosis, treatment and management of clinical conditions and physiological processes. The identification of novel devices and optimal use of existing devices which will lead to improved clinical outcomes and more effective patient management and safety is a key feature. The manuscript management system is completely online and includes a quick and fair peer-review system. Visit http://www. dovepress.com/testimonials.php to read real quotes from authors. 